\title{
Interdisciplinaridade e deficiência intelectual na educação especial: uma revisão sistemática integrativa
}

\author{
Interdisciplinarity and intellectual disability in special education: an integrative systematic review \\ Interdisciplinariedad y discapacidad intelectual en la educación especial: una revisión sistemática \\ integradora
}

Recebido: 07/02/2022 | Revisado: 14/02/2022 | Aceito: 23/02/2022 | Publicado: 04/03/2022

\author{
Débora Barbosa de Carvalho \\ ORCID: https://orcid.org/0000-0002-2039-8672 \\ Universidade Federal Fluminense, Brasil \\ E-mail: deb_nei@hotmail.com \\ Suzete Araujo Oliveira Gomes \\ ORCID: https://orcid.org/0000-0001-7130-8254 \\ Universidade Federal Fluminense, Brasil \\ E-mail: suzetearaujo@id.uff.br
}

\begin{abstract}
Resumo
O crescente número de alunos com deficiência intelectual matriculados nas escolas regulares desafia os profissionais da educação quanto à adequação do currículo escolar e da escola, para que estes estudantes tenham condições de desenvolver suas funções intelectuais e adaptativas, de acordo com suas necessidades individuais. A presente pesquisa pretendeu realizar uma Revisão Sistemática Integrativa de artigos sobre Educação Especial, com foco na Deficiência Intelectual, Interdisciplinaridade, e Formação de Professores. A associação dos dois termos, interdisciplinaridade e deficiência intelectual, são os eixos norteadores desta revisão sistemática integrativa. Este estudo analisou, em um primeiro momento, artigos do ano de 1980 a 2021, na Biblioteca Virtual do Portal de Periódicos CAPES, utilizando os seguintes descritores: Deficiência Intelectual, Interdisciplinaridade e Formação de Professores. Um total de 275 artigos foram encontrados, onde de acordo com os critérios de inclusão e exclusão, 16 artigos foram selecionados para leitura na íntegra. O resultado leva a concluir que o trabalho interdisciplinar, apesar de ser reconhecido por vários teóricos como uma ferramenta metodológica que poderia colaborar para uma aprendizagem significativa dos alunos da educação especial, ainda precisa ser reconhecida como elemento facilitador da aprendizagem entre os profissionais da educação. $\mathrm{O}$ trabalho interdisciplinar não se efetiva na prática, é necessário que os educadores se apropriem desta metodologia principalmente na educação especial.
\end{abstract}

Palavras-chave: Interdisciplinaridade; Deficiência intelectual; Formação de professores; Educação especial; Revisão sistemática integrativa.

\begin{abstract}
The crescent number of students with intellectual deficiency registered in regular schools challenges education professionals regarding to adequacy of scholar's curriculum and the school itself, in so that students have conditions to develop their intellectual and adaptational functions, according to their individual needs. This present research intended to accomplish one systematic revision of special education articles, focused on intellectual deficiency, interdisciplinarity and teacher training. The association of the two terms, interdisciplinarity and intellectual deficiency, are the guiding axis of this integrative systematic review. This study analyzed, at first moment, articles from 1980 to 2020, at Periodic Portal Virtual Library from CAPES using the following keywords: Intellectual Deficiency, Interdisciplinarity and TeacherTraining. A total of 275 articles were found according to inclusion and exclusion criterions, in which 16 articles were selected for full reading. The result leads to conclude that the interdisciplinary work, despite being recognized by several theorists as a methodological tool that could collaborate for one meaningful learning of special education students, still needs to be recognized as one learning facilitating element between educational professionals. The interdisciplinary work is not effective in practice, it is necessary that educators take ownership of this methodology, mostly in special education.
\end{abstract}

Keywords: Interdisciplinarity; Intellectual deficiency; Teacher training; Special education; Integrative systematical review.

\section{Resumen}

El creciente número de estudiantes con discapacidad intelectual matriculados en escuelas regulares desafía a los profesionales de la educación en cuanto a la adecuación del currículo escolar y la escuela, para que estos estudiantes sean capaces de desarrollar sus funciones intelectuales y adaptativas, de acuerdo con sus necesidades individuales. 
Esta investigación tuvo como objetivo realizar una Revisión Sistemática Integrativa de artículos sobre Educación Especial, con foco en Discapacidad Intelectual, Interdisciplinariedad y Formación Docente. La asociación de los dos términos, interdisciplinariedad y discapacidad intelectual, son los principios rectores de esta revisión sistemática integradora. Este estudio analizó inicialmente artículos de 1980 a 2020, en la Biblioteca Virtual del Portal de Revistas CAPES, utilizando los siguientes descriptores: Discapacidad Intelectual, Interdisciplinariedad y Formación Docente. Se encontraron un total de 275 artículos, donde de acuerdo con los criterios de inclusión y exclusión, se seleccionaron 16 artículos para lectura completa. El resultado lleva a la conclusión de que el trabajo interdisciplinario, a pesar de ser reconocido por varios teóricos como una herramienta metodológica que podría contribuir a un aprendizaje significativo de los estudiantes de educación especial, aún necesita ser reconocido como un facilitador del aprendizaje entre los profesionales de la educación. El trabajo interdisciplinario no es efectivo en la práctica, es necesario que los educadores se apropien de esta metodología, especialmente en educación especial.

Palabras clave: Interdisciplinariedad; Discapacidad intelectual; Formación docente; Educación especial; Revisión sistemática integrativa.

\section{Introdução}

O termo interdisciplinaridade foi criado em 1937, pelo sociólogo alemão, Louis Wirtz, sendo conceituado como tudo aquilo que se realiza com a cooperação de várias disciplinas interligadas e com relações definidas, que evitam desenvolver as suas atividades de forma isolada, dispersa ou fracionada. O movimento surgiu na Europa, principalmente na França e Itália em meados da década de 1960, justamente na época em que os estudantes europeus reivindicavam um novo estatuto de universidade e de escola. Dessa forma, a interdisciplinaridade nasceu com a proposta de se evitar a fragmentação no ensino, permitindo um olhar mais amplo na formação dos alunos que buscavam um ensino sintonizado com questões sociais, políticas e econômicas, ampliando conhecimentos mais contextualizados com a realidade (Fazenda, 1994; 1998).

No Brasil, a discussão sobre interdisciplinaridade chegou entre 1968 e 1971, como produto de reformas educacionais, cuja primeira discussão significativa sobre o assunto foi elaborada por Japiassú (1976), que abordava como condição para a definição dessa metodologia um tipo de profissional emancipador, com uma "nova consciência" e utilização de uma "nova pedagogia", baseada, principalmente, na comunicação. Para o autor, a interdisciplinaridade caracteriza-se pela intensidade das trocas entre os especialistas e pelo grau de integração real das disciplinas no interior de um mesmo projeto. No entanto, a alienação e o descompasso no trato das questões primordiais da interdisciplinaridade por parte dos educadores da época, somadas a pouca informação teórica e conceitual, levaram a estagnação do conhecimento da prática interdisciplinar durante muito tempo (Fazenda, 2003).

Na década de 1990 o movimento da interdisciplinaridade no Brasil reapareceu como possibilidade de um projeto interdisciplinar na educação. No final do século XX, a educação interdisciplinar aparece como a palavra-chave, motivando novas formas de pensar, novos métodos de ensino e nova concepção de escola (Fazenda, 2003).

Segundo Gadotti (2004), a proposta da interdisciplinaridade na educação é sair da "zona de conforto" e buscar novas alternativas que fujam da pedagogia tradicional, proporcionando ao aluno um ensino globalizante e integral, facilitando a aprendizagem na medida que, o conteúdo é trabalhado nas outras áreas de conhecimento dentro das especificidades de cada disciplina. Surgindo como uma forma de superar a discriminação, a exclusão e a descontextualização entre áreas de conhecimento, relacionando-as para uma melhor compreensão da realidade.

Diante de tantas mudanças que vem ocorrendo no mundo com relação as novas culturas que estão sendo inseridas, principalmente, das tecnologias, que vem facilitando as comunicações, relacionamentos sociais, econômicos, políticos, culturais etc, a própria sociedade exige das pessoas novas habilidades e competências. Portanto, investir em uma nova postura nas práticas educacionais é uma necessidade que o mundo vem exigindo dos profissionais da educação (Nóvoa, 2019). A interdisciplinaridade, vem de encontro a essa mudança de atitudes, propondo ações que visam uma educação contextualizada e significativa. De acordo com Vilela et al., (2003): 
A interdisciplinaridade é considerada uma inter-relação e interação das disciplinas a fim de atingir um objetivo comum. Nesse caso, ocorre uma unificação conceitual dos métodos e estruturas em que as potencialidades das disciplinas são exploradas e ampliadas. Estabelece-se uma interdependência entre as disciplinas, busca-se o diálogo com outras formas de conhecimento e com outras metodologias, com objetivo de construir um novo conhecimento. Dessa maneira a interdisciplinaridade se apresenta como resposta à diversidade, à complexidade e à dinâmica do mundo atual (Vilela et al., 2003, p. 529).

O professor exerce um grande poder nas formas que busca a aprendizagem dos seus alunos. É ele que irá mediar estas interações e oportunizar as aprendizagens. É ele que deverá buscar dentro das formações continuadas, novas teorias e novas formas de ensinar. Segundo Macedo (2004/2005, p. 17), "a escola de hoje deve mudar a visão que a de ontem construiu sobre si mesma, sem esquecer em sua crítica aquilo que continua valioso". E de acordo com Fernandes (2018):

Nesse diapasão, a Interdisciplinaridade se apresenta como uma solução. Esse modelo usa da interação de disciplinas aparentemente distintas, realizada de maneira complementar ou suplementar, como forma de possibilitar o desenvolvimento de um saber crítico-reflexivo, sendo este último o objetivo primordial desse novo sistema. Ademais, busca possibilitar um diálogo entre as disciplinas, relacionando-as entre si para uma compreensão diferenciada da realidade (Fernandes, 2018. p. 102).

Portanto, há necessidade de se explorar com mais cuidado a questão da metodologia do trabalho interdisciplinar, bem como a forma mais adequada de proceder-se a formação dos profissionais que efetivem a interdisciplinaridade, principalmente na educação especial. Dessa forma, o trabalho interdisciplinar é um caminho que pode colaborar no atendimento às necessidades cognitivas dos alunos com deficiência, em especial a Deficiência Intelectual (D.I.).

\section{Interdiscilinaridade $X$ Contexto da inclusão escolar}

O trabalho interdisciplinar é uma forma de dar um novo sentido às práticas pedagógicas dos professores da educação especial, na medida que colaboram na aprendizagem de crianças e jovens com deficiências, pois é uma proposta que busca um diálogo entre as áreas de conhecimento e colabora para o desenvolvimento das potencialidades e habilidades dos alunos.

Neste contexto, há necessidade da integração de uma equipe multidisciplinar: alunos, família, profissionais da educação para que essas trocas interdisciplinares, favoreçam positivamente o progresso da aprendizagem e da autonomia desse público alvo da educação especial. No entanto, a interdisciplinaridade é um desafio diário entre os profissionais envolvidos, pois deve haver o momento do diálogo, da troca, do planejamento, do envolvimento, onde nem sempre esse espaço é consolidado, mas deve ser construído, tendo como objetivo a realidade contextualizada por todas as áreas do conhecimento (Fazenda, 2008; Camas et al., 2021).

De acordo com Batista et al., (2004), "assim como o conceito de interdisciplinaridade vem sendo discutido desde os anos 70, buscando-se um consenso conceitual, debate-se sobre o mesmo tema sem que as práticas interdisciplinares seja uma proposta que aconteça dentro dos espaços educacionais com frequência. O mesmo cenário tem sido apresentado no que tange às práticas pedagógicas e políticas públicas que viabilizem a inclusão no sentido íntegro do conceito delineado historicamente até aqui, ou seja, o esforço de todas as áreas do conhecimento para a inclusão de todos que possam ser beneficiados por esta proposta no ensino regular". Ainda, segundo Batista et al., (2004), "a integração privilegia o aluno portador de necessidades educativas especiais, dividindo com ele a responsabilidade da inserção, enquanto a inclusão tenta avançar, exigindo da sociedade essa inserção".

Nesse sentido, os termos integração e inclusão se diferenciam quando falamos sobre a inclusão de alunos com deficiências na educação, já que a palavra integração nesse contexto divide com o aluno com deficiências a inserção no ambiente educacional, enquanto a inclusão vai mais além, uma vez que exige da sociedade condições para que ele seja incluído, pois não basta estar dentro da escola, deve haver condições para prover esse atendimento, de forma que o aluno não 
se sinta excluído. Pensar a educação especial sob o contexto da interdisciplinaridade e da intersetorialidade, é reconhecer a necessidade de aumentar a superfície de contato com outras disciplinas e saberes (Nocinovic \& Rodrigues, 2020).

O presente trabalho não tem objetivo de conceituar questões históricas sobre a inclusão, mas é necessário identificar atitudes e posicionamentos que permeavam e permeiam a inclusão dentro dos espaços educacionais, a fim de que possamos eliminar as barreiras que ainda impedem a inclusão efetiva desses alunos com deficiências na escola. Dessa forma, a intenção é refletir sobre o significado de inclusão e de interdisciplinaridade como propostas que são caracterizadas pelo trabalho coletivo, porém, com pouca produção registrada sobre experiências e documentos teóricos que indicam à associação dos dois termos nas propostas pedagógicas voltadas à inclusão escolar (Glat et al., 2003).

Diante dos fatos citados e percebendo a necessidade do aumento de estudos sobre Interdisciplinaridade, Educação Especial e Formação de professores, neste trabalho realizamos uma revisão integrativa que consiste de um levantamento, comparação e discussão dos dados existentes na literatura com o objetivo de investigar o que foi estudado sobre o tema, visando responder à pergunta focada no PICO: A interdisciplinaridade é aplicada na Educação especial? e dessa forma, contribuir para o desenvolvimento de novas pesquisas na área.

\section{Metodologia}

Neste estudo, a coleta de dados sobre o tema, Interdisciplinaridade e Educação especial, foi realizada a partir de fontes secundárias, por meio de levantamento bibliográfico e baseado na experiência vivenciada pelas autoras por ocasião da realização de uma revisão integrativa.

Para isso, realizamos os 5 passos metodológicos para uma revisão integrativa baseados e atualizados por Whittemore e Knafl (2005) e Galvão et al., (2014), respectivamente: Pergunta norteadora ou Identificação do Problema, Estratégia de busca ou Amostragem na literatura, Coleta de dados, Análise dos dados e Apresentação da revisão integrativa seguindo o protocolo PRISMA Statements (Preferred Reported Items for Sistematic Reviewsand Meta-analyses) (Page et al., 2021).

A pesquisa e o processo de seleção dos artigos foram conduzidos pelas autoras e quando ocorreu diferença na escolha dos artigos, após a leitura, estas entraram em consenso. A síntese dos dados extraídos dos artigos foi realizada a partir de uma abordagem qualitativa descritiva (Pereira et al., 2018), possibilitando observar, descrever e classificar os dados, com o intuito de reunir o conhecimento produzido sobre o tema explorado nesta revisão.

\section{Pergunta norteadora ou Identificação do problema}

Esta revisão foi conduzida por uma pergunta-guia - A interdisciplinaridade é aplicada na Educação especial? estruturada pela estratégia que auxilia na construção de uma pergunta de pesquisa e busca de evidências, como PICO (acrônimo para População ou Paciente, Intervenção, Comparação e "Outcomes" (desfecho)) onde os critérios de inclusão foram descritos de acordo com a População (P): alunos com Deficiência Intelectual; Intervenção (I): interdisciplinaridade na educação especial; Comparações (C): interdisciplinaridade e formação de professores; e Resultados (O): educação especial.

\section{Estratégia de busca ou amostragem na literatura}

As pesquisas foram realizadas utilizando o Portal de Periódicos CAPES, no período de 1980 a 2021, investigando as seguintes bases de dados: SciELO, Eric, OneFile (GALE), SAGE Journals, SAGE Publications, Taylor \& Francis online Journals e MEDLINE/PubMed.

As palavras utilizadas na busca dos artigos seguiram os termos DeCS - (Descritores em Ciências da Saúde) desenvolvido pelo MeSH (The Medical SubjectHeadings) da US National Library of Medicine. As pesquisas foram conduzidas utilizando os seguintes descritores como termos de busca e os operadores booleanos: Interdisciplinary work 
education AND disability intellectual NOT disability mental. Todos os artigos encontrados pela estratégia de busca foram coletados através do gerenciador de referências acadêmicas, o software Zotero (versão 5.0.80).

\section{Critérios de inclusão e exclusão}

Os termos foram pesquisados no título e no resumo ou abstract de acordo com o Guia Cochrane (Garritty et al., 2020), ou no texto completo quando o título e o resumo não esclareceram o conteúdo do artigo. Também foram utilizados, filtros que incluíssem apenas artigos científicos, em um dos seus campos de texto (resumo, corpo do texto, título e referências), em um ou mais descritores.

Foram excluídos capítulos de livro, cartas editoriais e artigos duplicados. Posteriormente, realizamos uma seleção de artigos através da leitura de título, palavras-chave e resumo, selecionando apenas artigos que mencionaram os termos interdisciplinaridade, deficiência intelectual e formação de professor na Educação Especial.

\section{Análise e Coleta de dados}

Após a aplicação dos critérios de inclusão e exclusão, o procedimento de análise dos artigos consistiu na leitura completa dos artigos obtidos, considerando apenas aqueles que se encontravam alinhados com o objetivo da pesquisa. A coleta de dados se baseou nos conceitos embasadores sobre Interdisciplinaridade, Deficiência Intelectual e Formação de professores na Educação especial, além do registro das informações para obtenção dos seguintes indicadores, os quais compõem o Quadro 1: título, temática estudada e ano de publicação.

\section{Síntese de Dados}

Após a leitura completa dos artigos selecionados, para ilustrar a inclusão e exclusão de artigos, utilizamos o diagrama de Prisma (Figura 1). Este diagrama trata-se de um esquema ilustrando o processo de seleção e triagem dos artigos. Para isso, utilizamos o software gratuito online de acesso por navegador de internet draw.io v. 10.6 .5 (disponível em: https://www.draw.io), (Page et al., 2021).

\section{Resultados}

O resultado final das buscas nas bases de dados gerou um total de 275 artigos, sendo encontrados: 75 no Eric; 70 no OneFile (GALE); 41 no SAGE Journals; 34 no Taylor \& Francis Online; 33 no SAGE Publications; 01 no SciELO e 21 no MEDLINE/PubMED. Ao realizar as confluências entre os descritores referente à pesquisa, encontramos os seguintes resultados, considerando os anos compreendidos entre 1980 a 2021 (Tabela 1).

Tabela 1: Confluência entre os Descritores nas Bases de Dados.

\begin{tabular}{|c|c|}
\hline Eixos & PORTAL DE PERIÓDICO CAPES \\
\hline Interdisciplinary work in education AND intellectual disability & 98 artigos \\
\hline Interdisciplinarity in education AND intellectual disability & 01 artigo \\
\hline Interdisciplinary work education AND disability intellectual NOT disability mental & 275 artigos \\
\hline
\end{tabular}


Dos 275 artigos encontrados, foram excluídos 119 artigos duplicatas, totalizando 156 artigos de pesquisa, dos quais 126 foram excluídos, incluindo artigos de revisão, restando 30 artigos que foram selecionados para leitura completa. Após todo processo de pesquisa, triagem e seleção dos artigos, foi elaborado um diagrama de Prisma (Figura 1) considerando somente o descritor Interdisciplinary work education AND disability intellectual NOT disability mental, tendo em vista a maior quantidade de artigos encontrados. De acordo com a leitura completa dos 30 artigos obtidos, consideramos que apenas 16 artigos se encontravam alinhados à pesquisa.

Figura 1: Fluxograma de seleção de estudos sobre Interdisciplinaridade e Deficiência Intelectual na Educação Especial para inclusão na revisão bibliográfica.

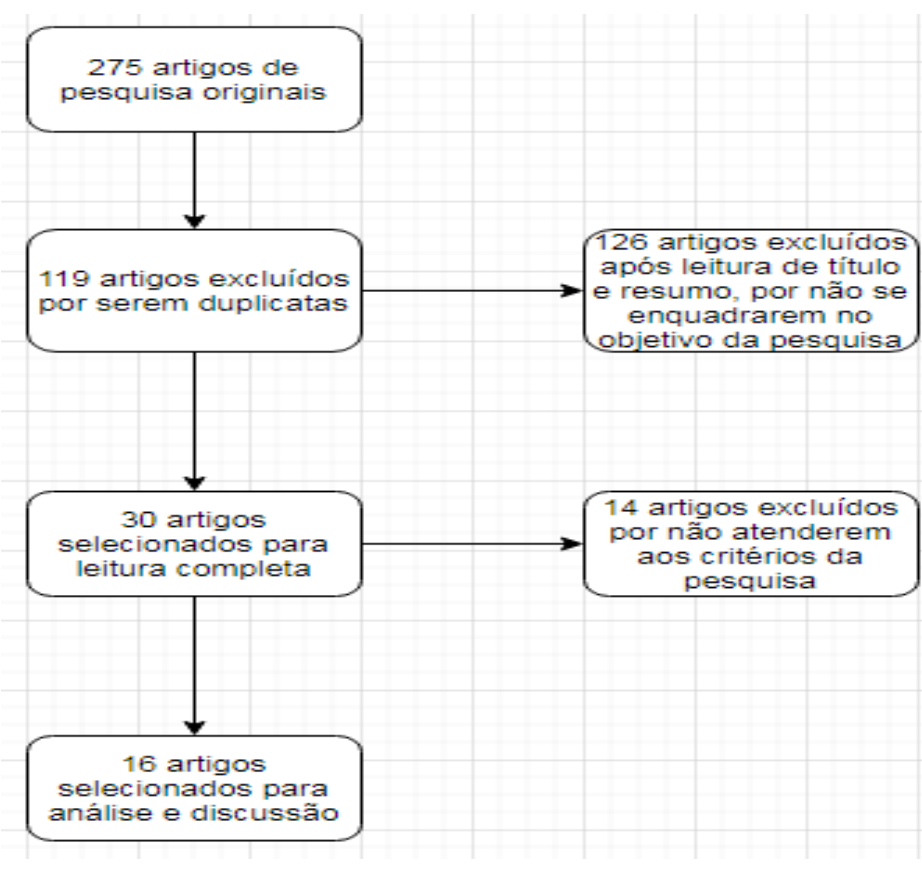

Fonte: Autores.

Software online de acesso por navegador de internet draw.io v. 10.6.5 (disponível em http:///www.draw.io).

\section{Análise dos dados}

Ao analisarmos a cronologia dos 16 artigos selecionados, observamos que a maioria dos artigos são recentes. Sendo apenas três artigos publicados entre os anos de 1995 e 1996. Os demais foram publicados a partir de 2001. A maior parte das publicações, concentraram-se entre os anos de 2011 a 2018 (Quadro 1). 
Quadro 1: Artigos selecionados para análise, considerações e conclusões:

\begin{tabular}{|c|c|c|}
\hline ARTIGOS & CATEGORIA DE ARTIGOS & ANO \\
\hline Addressing learning differences rights from the start & Interdisciplinaridade & 1995 \\
\hline The resource center: hub of inclusive activities & Interdisciplinaridade & 1996 \\
\hline Creating a Responsive Curriculum for Inclusive Schools & Interdisciplinaridade & 1996 \\
\hline The value of critical perspectives in teacher education & Formação de Professores & 2001 \\
\hline $\begin{array}{l}\text { The inclusive school strategies to deal with it: the } \\
\text { curricular adaptations }\end{array}$ & Interdisciplinaridade & 2010 \\
\hline $\begin{array}{c}\text { De volta à teoria da curvatura da vara: a deficiência } \\
\text { intelectual na escola inclusiva }\end{array}$ & DeficiênciaIntelectual & 2011 \\
\hline $\begin{array}{l}\text { Student socialization in interdisciplinary doctoral } \\
\text { education }\end{array}$ & Interdisciplinaridade & 2011 \\
\hline $\begin{array}{l}\text { An Examination of Learning Formats on Interdisciplinary } \\
\text { Teamwork Knowledge, Skills, and Dispositions }\end{array}$ & Interdisciplinaridade & 2011 \\
\hline $\begin{array}{l}\text { Learning outcomes and } \\
\text { inclusive education of students with Intellectual disabiliti } \\
\text { es }\end{array}$ & Deficiência Intelectual & 2012 \\
\hline $\begin{array}{c}\text { Ishodiučenja i } \\
\text { inkluzivnaedukacijaučenikasintelektualnimteškoćama } \\
\text { (Língua Croata, traduzido através do google tradutor e } \\
\text { significa: Resultados de aprendizagem e educação } \\
\text { inclusiva de alunos com deficiências intelectuais) }\end{array}$ & Deficiência Intelectual & 2013 \\
\hline $\begin{array}{c}\text { Cuando el proyecto se hace método: nuevas perspectivas } \\
\text { para la investigación socioeducativa en red. }\end{array}$ & Deficiência Intelectual & 2015 \\
\hline Secondary schools included: a literature review & Formação de Professores & 2015 \\
\hline $\begin{array}{c}\text { Caracterização da avaliação da aprendizagem nas salas de } \\
\text { recursos multifuncionais para alunos com deficiência } \\
\text { intelectual }\end{array}$ & Deficiência Intelectual & 2016 \\
\hline $\begin{array}{l}\text { Becoming dishuman: thinking about the human through } \\
\text { disability }\end{array}$ & DeficiênciaIntelectual & 2016 \\
\hline $\begin{array}{c}\text { Experiences of continuing teachers education: } \\
\text { Possibilities for effective inclusion of students with } \\
\text { intellectual disabilities }\end{array}$ & Formação de Professores & 2017 \\
\hline $\begin{array}{c}\text { Promoting Inclusive Education: The Role of Teachers' } \\
\text { Competence and Attitudes }\end{array}$ & Formação de Professores & 2018 \\
\hline
\end{tabular}

Fonte: Autores.

Após leitura completa, analisamos os artigos selecionados, de acordo com o escopo desta pesquisa, sendo então 4 artigos para formação de professores; 6 artigos para D.I. e 6 artigos para Interdisciplinaridade (Quadro 1).

Em relação à classificação dos artigos, segundo os temas delimitados em nossos critérios de pesquisa (Tabela 1), podemos considerar que não foram encontrados artigos onde os três temas: Interdisciplinaridade, Formação de professores e Deficiência Intelectual (D.I.) encontravam-se citados no mesmo material de leitura. Entretanto, observamos que o tema Formação de professores permeava os artigos selecionados e estava atrelado à interdisciplinaridade e à D.I. (Hardin et al., 1996; Udvari-Solner et al., 1996; Beyer, 2001; Heredero, 2010; Boden et al, 2011; Ivey et al., 2011; Skills et al., 2011; Gómez et al., 2015; Anache et al., 2016; Pletsch et al., 2017; Cate et al., 2018).

Além disso, observamos também que o termo Interdisciplinaridade foi raramente abordado em alguns artigos relacionados à inclusão (Heron et al., 1995; Bezerra et al., 2011; Žiljak, 2013; Goodley et al., 2016; De Vroye et 1., 2016).

A dificuldade de se trabalhar a Interdisciplinaridade está, principalmente, no modelo educacional vigente, que trabalha separadamente cada disciplina. Nos 06 (seis) artigos encontrados sobre o tema Interdisciplinaridade, os autores buscaram melhores condições e oportunidades de aprendizagens significativas (Heron et al., (1995); Hardin et al., (1996); Solner et al., (1996); Heredero (2010); Boden et al., (2011); Ivey et al., (2011). 


\section{Discussão}

Um caminho coerente para tentar esclarecer controvérsias sobre um determinado tema, é apoiar-se em estudos de qualidade (Galvão et al., 2014). Diante disso, ao escolher pesquisar sobre Interdisciplinaridade e Educação especial, optamos por fazer uma revisão integrativa, pois de acordo com Whittemore et al., (2005) é um método que proporciona a síntese do conhecimento sobre um tema ou questão, de maneira sistemática, ordenada, abrangente e permite a incorporação da aplicabilidade de resultados de estudos significativos na prática.

Passamos, então, a falar sobre a interdisciplinaridade na educação especial, que junto à Deficiência Intelectual, são o foco desta pesquisa. Sabemos que trabalhar de forma interdisciplinar não é fácil, pois envolve interações, diálogos com seus pares, planejamentos, apropriações de conhecimentos que podem ir além do que o professor domine e a colaboração de toda a equipe diretiva. Segundo Gattás et al., (2005), “o estudo da interdisciplinaridade é ainda incipiente, não oferecendo segurança teórica e metodológica para àqueles que se sentem atraídos para desenvolver um trabalho dessa envergadura". Nacinovic et al., (2020), apontam para a hegemonia do campo biológico, especificamente da medicina sobre a educação especial.

Cumpre ressaltar que a interdisciplinaridade é baseada, antes de tudo, na relação entre pessoas, o que implica nos problemas contidos em todas as relações interpessoais, pois somos diferentes, os pensamentos divergem, porém, é na diversidade, no respeito e na humildade, que podem nascer grandes projetos que poderão favorecer a aprendizagem dos alunos com deficiência intelectual e os demais alunos, com deficiência ou não (Murca, 2019; Neves et al., 2019; Mattos et al., 2021). No entanto, mesmo diante do consenso de vários teóricos a respeito da relevância da interdisciplinaridade como ferramenta para evolução dos profissionais e, principalmente, dos educandos, destinatários de tais projetos interdisciplinares, ainda é possível observar que não foram produzidos grandes avanços que poderiam acarretar em reflexos significativos a respeito da interdisciplinaridade no cotidiano educacional.

Nesta revisão sistemática integrativa, os artigos analisados (Quadro 1) destacaram que os alunos com Deficiência Intelectual (D.I.) estão se matriculando nas escolas de ensino regular. Entretanto, há uma enorme barreira entre estar inserido em um espaço da sala de aula e estar incluído em uma escola que propõe práticas democráticas, igualitárias e inclusivas. O que se percebe, é que as escolas não estão preparadas para receber os alunos da educação especial, pois ainda estão revestidas de um preconceito de que os educandos com deficiência, principalmente, os que apresentam D.I., ainda são concebidos como alunos com atraso mental e dessa forma, sua condição é compreendida como inferior aos demais. Sendo assim, estão fadados ao fracasso na escola, pois não têm condições de aprender como os outros alunos que não apresentam deficiências (Kupfer et al., 2000; Ferreira, 2007). No entendimento de Mantoan (2004), a inclusão de alunos com necessidades educacionais especiais é um movimento com muitas polêmicas.

Sob outro aspecto, a interdisciplinaridade, bem como a inclusão, é baseada, antes de tudo, na relação entre pessoas, o que implica nos problemas contidos em todas as relações interpessoais. Para Vilela e Mendes (2003), a interdisciplinaridade é considerada uma inter-relação e interação das disciplinas a fim de atingir um objetivo comum. Estabelece-se uma interdependência entre as disciplinas, busca-se o diálogo com outras formas de conhecimento e com outras metodologias, com objetivo de construir um novo conhecimento.

Com relação aos professores, não basta somente ter atendimento educacional especializado, ou seja, um professor da sala de recursos para atender esses alunos da educação especial. Ao professor do ensino regular, deve-se prover formações adequadas e específicas para o trabalho inclusivo, buscando novas formas de ensino para incluir efetivamente o aluno com deficiência e não apenas, integrá-lo na turma (Nacinovic et al., 2020).

Dentro deste contexto, a interdisciplinaridade surge como uma importante prática educacional onde o conhecimento deixa de ser fragmentado para tornar-se contextualizado, através da interação entre as disciplinas, tornando o ensino mais democrático, na medida que se preocupa com as diversidades de cada aluno e através das ações e intervenções pedagógicas 
interdisciplinares, proporciona a ampliação das habilidades cognitivas, principalmente dos alunos com D.I.

A fragmentação do conhecimento não colabora com o desenvolvimento das potencialidades dos alunos com deficiência intelectual. Pelo contrário, facilitam as desigualdades sociais e a exclusão. Fonseca (1995), acredita que é preciso preparar todos os professores, com urgência, para se obter sucesso na inclusão, através de um processo de inserção progressiva; assim eles poderão aceitar e relacionar-se com seus diferentes alunos e, consequentemente, com suas diferenças e necessidades individuais.). Portanto, a formação continuada ou em serviço é fundamental para o professor se apropriar de instrumentos que vão lhe propiciar novas aquisições e competências, favorecendo a superação do medo e da insegurança na sua prática pedagógica.

De acordo com Rodrigues (2012), "É preciso reconhecer que o Brasil tem uma base de sustentação legal que deveria contribuir para que as mudanças educacionais acontecessem num processo crescente de introdução de novos conhecimentos e novas práticas". Portanto, o professor deve buscar essas mudanças na sua formação continuada e introduzir novas práticas pedagógicas, de forma que contribua para a inclusão efetiva do aluno com deficiência na escola.

Há também grandes barreiras que impedem os profissionais da educação em construir uma postura interdisciplinar, devido a alguns fatores. Entre eles, podemos citar a falta de tempo de se planejar coletivamente ou mesmo pela dificuldade de interagir com outras disciplinas. Para Santomé (1998), "a interdisciplinaridade tem sua razão de ser na busca de uma grande teoria, uma nova etapa do desenvolvimento das ciências caracterizado por uma reunificação do saber em um modelo que possa ser aplicado a todos os âmbitos atuais do conhecimento". Para outros, o caminho de interdisciplinaridade é provocado pela dificuldade, que se torna mais evidente a cada dia, de delimitar as questões que são objetos deste ou daquele campo de especialização do saber. Entretanto, ainda é vista com cautela por muitos profissionais da educação, por entenderem que as atividades interdisciplinares acabam com a disciplina e as áreas do conhecimento curricular. Nesse sentido, ainda "[...] estamos bastante divididos entre um passado que negamos, um futuro que vislumbramos e um presente que está muito arraigado dentro de nós" (Fazenda, 2003).

Portanto, se faz necessária a formação profissional para a inclusão e a predisposição para a interdisciplinaridade, pois esta não tem metodologias prontas, ela é criada e (re) criada diariamente entre os profissionais da educação que percebem que a mudança nas práticas pedagógicas é necessária diante de tanta diversidade e do respeito às diferenças entre cada pessoa. Por ser um trabalho que exige interações, ousadia e coletividade, possui pouca produção registrada sobre experiências interdisciplinares inclusivas nas propostas pedagógicas das escolas.

Mudanças no campo da formação docente para a educação inclusiva podem contribuir de maneira significativa para que experiências bem-sucedidas não sejam mais exceções no nosso cenário educacional e sim a norma de uma educação democrática (Rodrigues, 2012; Nóvoa, 2019) e passível de serem divulgadas.

\section{Considerações Finais}

De acordo com os artigos selecionados e lidos, a deficiência intelectual ainda sofre com práticas excludentes e segregadoras, pois as escolas não atendem às necessidades cognitivas desses alunos e não apresentam metodologias de trabalho ou currículos diferenciados, necessários à aprendizagem.

Em adição, observamos que o trabalho interdisciplinar não se efetiva de fato, pois falta um trabalho coletivo e uma ação pedagógica, onde a fragmentação da prática na e da escola ainda é uma realidade inegável. Mesmo diante do consenso de vários teóricos sobre a importância do trabalho interdisciplinar, faltam diálogos, planejamentos, interações, intenções, flexibilizações por parte dos profissionais da educação sem falar nos preconceitos e rivalidades que geram conflitos dentro da escola e dificulta a prática desta ferramenta de trabalho. Foi possível observar, na revisão literária, que não foram produzidos grandes avanços que poderiam acarretar reflexos significativos na educação especial a respeito da interdisciplinaridade no 
cotidiano educacional.

Ao analisar o processo de formação do professor, observamos que há necessidade de se buscar a formação continuada do professor/educador no contexto inclusivo. Além disso, observamos também que é de suma importância ter um sistema de ensino preparado para receber alunos com diferentes tipos de deficiências, entre elas, a deficiência intelectual. Deve-se adequar o ambiente escolar para recebê-los e preparar os profissionais que fazem parte da comunidade escolar, a fim de que estes consigam atendê-los em suas especificidades e habilidades.

Com esta Revisão Sistemática Integrativa, esperamos contribuir para repensarmos o que é necessário mudar ou melhorar para avançarmos em uma educação especial que atenda, com qualidade, às necessidades dos alunos com deficiências. E que outras contribuições literárias sejam realizadas para continuarmos avançando nessa temática tão propícia ao momento em que vivemos.

Nesse sentido, a Revisão Sistemática Integrativa relacionada à Interdisciplinaridade, Deficiência Intelectual e Formação de Professores na Educação Especial, vem contribuir para fomentar novas reflexões e ações para a prática docente privilegiando uma educação inclusiva de qualidade.

\section{Agradecimentos}

Agradecemos ao Curso de Mestrado Profissional em Diversidade e Inclusão (CMPDI) da Universidade Federal Fluminense.

\section{Referências}

Anache, A. A. \& Resende, D. A. R. (2016). Caracterização da avaliação da aprendizagem nas salas de recursos multifuncionais para alunos com deficiência intelectual. Revista Brasileira de Educação, 21(66), 569-591.

Batista, M. W. \& Enumo, S. R. F. (2004). Inclusão Escolar e Deficiência Mental. Estudos de Psicologia, 9(1), $101-111$.

Beyer, L. E. (2001). The Value of Critical Perspectives in Teacher Education. Journal of Teacher Education, 52(2), $151-163$.

Beyer, H. O. (2006). Da Integração Escolar à Educação Inclusiva: implicações pedagógicas. Mediação.

Bezerra, G. F. \& Araújo, D. A. C. (2011). De volta à teoria da curvatura da vara: a deficiência intelectual na escola inclusiva (Back to the stick's curvature theory: intellectual disabilities in inclusive school). Educar em Revista online, 27(2), 277-302.

Boden, D., Borrego, M., \& Newswander, L. K. (2011). Student socialization in interdisciplinary doctoral education. Higler Education, 62, 741-755.

Camas, N. P. V., Lambach, M., \& Souza, F. R. A. (2021). Interdisciplinaridade e Alfabetização Científica: um ensaio sobre os dois lados da mesma moeda. Ensino Em Re-Vista, 28, 1-23.

Cate, I. M. P., Markova, M., \& Krischler, M. (2018). Promoting Inclusive Education: The Role of Teachers' Competence and Attitudes. Insights into Learning Disabilities, 15(1), 49-63.

De Vroey, A., Struyf, E., \& Petry, K. (2016). Secondary schools included: a literature review. International Journal of Inclusive Education, 20(2), 109-135.

Fazenda, I. C. A. (1994). Interdisciplinaridade: História, teoria e pesquisa. Campinas: Papirus.

Fazenda, I. C. A. (1998). Didática e Interdisciplinaridade. Papirus.

Fazenda, I. C. A. (2003). Interdisciplinaridade: qual o sentido? Paulus.

Fazenda, I. C. A. (2008). Interdisciplinaridade e transdisciplinaridade na formação de professores. Revista do Centro de Educação e Letras, $10(1)$, 93-103.

Fernandes, A. M. M. (2018). Interdisciplinaridade no ensino e aprendizagem: novas perspectivas e desafios na atualidade. Id on Line: Revista Multidisciplinar e de Psicologia, 12(40), 101-115.

Ferreira, M. C. C. (2007). O desenvolvimento profissional do docente e a inclusão escolar de alunos com deficiência mental. ABPEE/FAPESP.

Fonseca, V. (1995). Educação Especial. Artes Médicas.

Gadotti, M. (2004). Pensamento Pedagógico Brasileiro. Ática.

Galvão, T. F.\& Pereira, M. G. (2014). Revisões sistemáticas da literatura: passos para sua elaboração. Epidemiologia e Serviços de Saúde, $23(1)$, $183-184$. 
Garritty, C., Gartlehner, G., Kamel, C., King, V.J., Nussbaumer-Streit, B., Stevens, A., Hamel, C., \& Affengruber, L. (2020). Cochrane Rapid Reviews, Interim Guidance from the Cochrane Rapid Reviews Methods Group, (1), 1-2.

Glat, R. \& Nogueira, M. L. L. (2003). Políticas Educacionais e a Formação de Professores para a Educação Inclusiva no Brasil. Comunicações, 10(1), 134141.

Gómez, J. A. C. \& Vale, R. F. (2015). Cuando el proyecto se hace método: nuevas perspectivas para la investigación socioeducativa en red. Pedagogia Social Revista Interuniversitaria, 26, 139-172.

Goodley, D. \& Runswick-Cole, K. (2016). Becoming dishuman: thinking about the human through disability. Discourse: Studies in the Cultural Politics of Education, 37(1), 1-15.

Hardin, D. E. \& McNelis, S. J. (1996). The Resource Center: Hub of Inclusive Activities. Handbook of Children with Special Health Care Needs. New York: Springer.

Heredero, E. S. (2010). The inclusive school strategies to deal with it: the curricular adaptations. Acta Scientiarum Education, 32 (2), 193-208.

Heron, E.\& Jorgensen, C. M. (1995). Addressing Learning Differences Right from the Start: the Inclusive School. Educational Leadership, 52(4), 56-58.

Ivey C. K. \& Reed E. (2011). An Examination of Learning Formats on Interdisciplinary Teamwork Knowledge, Skills, and Dispositions. Interdisciplinary Journal of Teaching and Learning, 1 (1), 43-55.

Japiassú, H. (1976). Interdisciplinaridade e patologia do saber. Imago.

Kupfer, M. C. M., \& Petri, R. (2000). “Por que ensinar a quem não aprende?”. Estilos da Clínica. Revista sobre a infância com problemas, (9), 109-117.

Macedo, L. (2004). Ensaios Pedagógicos: como construir uma escola para todos? Artmed.

Mantoan, M. T. E. (2003). Inclusão escolar: o que é? Por quê?? Como fazer? Moderna.

Mantoan, M. T. E. (2005). Inclusão é o privilégio de conviver com as diferenças. Moderna.

Mattos, S. M. N. \& Oliveira, K. F. (2021). Práticas docentes inovadoras e insurgentes: interdisciplinaridade e contextualização como possíveis caminhos. Ensino em Re-Vista, 28, 1-20.

Murca, J. G. (2019). Educação Inclusiva do deficiente intelectual: leitura e escrita. Revista Científica Multidisciplinar Núcleo do Conhecimento, 03(04), 137149.

Nacinovic, R.C. P. \& Rodrigues, M. G. A. (2020). Interdisciplinaridade e espaços dialógicos na educação inclusiva: encontros possíveis entre educação e saúde. Imagens da Educação, 10(2), 92-103.

Neves, L. R., Rahme, M. M. F., \& Ferreira, C. M. R. J. (2019). Política de educação especial e os desafios de uma perspectiva inclusiva. Porto Alegre: Educação e Realidade.

Nóvoa, A. (2019). Os professores e sua formação num tempo de metamorfose da escola. Educação e Realidade.

Page, M. J., McKenzie, J. E., Bossuyt, P. M., Boutron, I., Hoffmann, T. C., Mulrow, C. D., Shamseer, L., Tetzlaff, J. M., Akl, E. A., Brennan, S. E., et al. (2021). The PRISMA 2020 statement: An updated guideline for reporting systematic reviews. BMJ Open, 372(71), 1-9, http://dx.doi.org/10.1136/bmj.n71.

Pletsch, M. D., Araujo, D. F., \& Costa, M. F. L. (2017). Experiences of Continuing Teachers Education: Possibilities for Effective Inclusion of Students with Intellectual Disabilities. Periferia, 9(1), 290.

Rodrigues, S. M. (2012). Educação inclusiva e formação docente. Diversa.

Sampaio, R. F., \& Mancini, M.C. (2007). Systematic review studies: a guide for careful synthesis of the scientific evidence. Revista brasileira de fisioterapia, 11(1), 83-89.

Santomé, J. T. (1998). Globalização e interdisciplinaridade: o currículo integrado. Editora Artes Médicas Sul Ltda.

Sassaki, R. K. (1997). Inclusão: construindo uma sociedade para todos. WVA.

Sassaki, R. K. (2002). Terminologia sobre deficiência na era da inclusão. Revista Nacional de Reabilitação, 5(24), 6-9.

Skills, T. K., Ivey, C. K., \& Reed, E. (2011). An Examination of Learning Formats on Interdisciplinary. Journal of Teaching and Learning, 1(1), 43-55.

Stobäus, C.D. \& Mosqueira, J. J. M. (2004). Educação Especial: em direção à Educação Inclusiva.: EDIPUCRS.

Udvari-Solner, A. \& Thousand, J. S. (1996). Creating a responsive curriculum for inclusive schools. Remedial and Special Education, 17(3), 182-192.

Vilela, E. M. \& Mendes, I. J. M. (2003). Interdisciplinaridade e saúde: estudo bibliográfico. Revista Latino-Americana, 11(4), 525-531.

Whittemore, R. \& Knafl, K. (2005). The integrative review: updated methodology. Journal of Advanced Nursing, 52(5), 546-553.

Žiljak, O. (2012). Learning Outcomes and Inclusive Education of students with Intellectual Disabilities. Revija Socijalnu Politiku, 23(23), 81-92.

Žiljak, O (2013). Ishodiučenja i inkluzivnaedukacijaučenikasintelektualnimteškoćama. Agencijazastrukovnoobrazovanje i obrazovanjeodraslih. Primljeno: Svibanj. 